\title{
Longitudinal Sleep Patterns during Pubertal Growth: Four-year Follow-up
}

\author{
ISMET KARACAN, ${ }^{(25)}$ MICHAEL ANCH, JOHN I. THORNBY, MASAKO OKAWA, \\ AND ROBERT L. WILLIAMS \\ Department of Psychiatry, Baylor College of Medicine, Texas Medical Center, and Veterans Administration \\ Hospital, Houston, Texas, USA
}

\section{Extract}

There were little or no indications of differences in sleep outcomes between the sexes. Results indicate a disturbance of sleep on initial laboratory nights relative to later nights. The results reported here clearly document the persistence of these effects from year to year. For the most part, sleep characteristics during the 4 years immediately after onset of puberty appear to represent a typical phase in the gradual patterns of changes across all ages. Total sleep time decreased markedly from $560 \mathrm{~min}$ in age range $10-12$ to 424 $\mathrm{min}$ in age range $20-29$, with our puberty subjects at intermediate levels. Puberty subjects had an average of 2.5 awakenings/night in the first 2 years as compared with 1.2 / night in the last 2 years. The number of sleep stage shifts during the night laried around a constant mean value of approximately $37 /$ night throughout all ages. The number of rapid eve movement (REM) periods during the night decreased sharply for individuals from childhood $(6.9 /$ night $)$ through adolescence $14.0 /$ night), remaining constant thereafter. Percentages of the various sleep stages were fairls constant for individuals from age range 10-12 through age 30-39. Our puberty subjects had percentage profiles in near perfect agreement with the normal ontogenetic process. Jormative data suggest that slow wave sleep reaches a peak at some point during the teen years.

\section{Speculation}

Stability of sleep rather than its disruption may prove to be the rule rather the exception. Sleep may be refractori to developmental change. This may have surival value.

Marked changes in the biologic and psychologic states of a human individual are characteristic to the period of puberty. Although sleep patterns are known to follow gradual trends across all ages (21), during the relatively short period of puberty it might be expected that psychobiologic effects could reflect changes in sleep characteristics unpredicted by the gradual ontogenetic trends. Therefore, it is becoming increasingly apparent that a complete understanding of the regulation of physiologic systems requires a consideration of the role sleep plays in these systems.

The sensitivity of physiologic $(10,11)$ and psychologic $(9,12,20)$ changes to sleep makes this time particularly important in evaluating changes in the physiologic systems. On the other hand, sleep itself may be affected by changes in the physiologic or psychologic environment of the individual (1, 7. 14, 18). Indeed, the normal process of aging is associated with rather marked changes in the sleep pattern $(5,17,21)$, such that what is considered abnormal or disrupted sleep at one age may be within normal range limits for another age group.

Puberty is an important transitional period in the life cycle, but, despite the intense activity characteristic of this phase, little is known of the mechanisms of change or the effects on other systems. For example, changes indigenous to puberty may induce effects on sleep patterns. Sleep characteristics which change as a function of puberty may reflect an endogenous compensation in central nervous system activity. Alternatively, the sleep rhythm mechanism may be immune to such endogenous change, i.e., stability of the sleep pattern would be biologically adaptive to the species. If this were the case, plasticity of the sleep mechanism to endogenous change may be required in order to insure normal functioning of the individual. Resolution of these issues would provide additional information concerning the fundamental question in sleep research, i.e., its function. Further, it may help to clarify uncertainties concerning the mechanisms involved in heralding the onset of puberty in relation to sleep, if indeed there are any.

Electroencephalographic (EEG) sleep patterns have been studied as a function of age, from childhood through senescence (21), thereby encompassing the pubertal phase. A general decrease in sleep time was found with increasing age, along with systematic changes in the percentages of and latencies to the various sleep stages. The effects of puberty per se on sleep parameters were masked by the grouping of subjects into 3-year and wider age ranges.

One study (22) focused on puberty, comparing sleep patterns among three groups of male subjects: preadolescent (8-11 years), pubertal (13-15 years). and young adult (21-31 years). The percentage of stages 2 and 4 in the two younger groups differed significantly from the young adult group, with younger males having more stage $4(18 \%$ vs. $13 \%)$ and less stage $2(44 \%$ vs. $49 \%)$ than young adults.

Boyar et al. (2) have shown convincingly that changes do take place in physiologic systems during puberty. They found significantly higher spurts of luteinizing hormone $(\mathrm{LH})$ secretion during sleep than during waking during puberty. Prepubertal and adult males did not demonstrate any significant difference between waking and sleep LH secretion.

Reports comparing the sleep characteristics among age groups have generally neglected the period of puberty. There are no literature references, for example, indicating pubertal sleep effects in females.

The present study was a 4-year longitudinal sleep evaluation beginning with pubertal onset of normal male and female subjects, and comparisons are made with sleep characteristics of other preand postpubertal groups of subjects. An evaluation was also made of the sleep-disturbing effects of the first laboratory night during each of the 4 years of the study.

\section{METHODS}

Subjects consisted of 7 male and 10 female high school students in good health, ranging in age from 12.5 to 15.8 (mean 14.2) for the males and from 14 to 15.8 (mean 14.9) for the females. Originally there were 10 male subjects but 3 were unable to complete all 4 study years. Selection was based on chronologic age, bone age (23), and pubertal growth, utilizing criteria defined by Donovan and Van Der Werff Ten Bosch (4). Mean bone ages were 13.2 years for males and 13.8 years for females. All subjects had a 
physical examination and psychiatric evaluation, and were free of any signs or symptoms of physical abnormality or psychopathology. The first study night occurred approximately 6 months after the onset of puberty for all subjects. Informed consent was obtained from all subjects.

Some of the puberty subjects used in this study (four males and eight females) were also contained in the 13-15 year age group in a report of normative ontogenetic sleep patterns by Williams et al. (21). Subjects included in the normative study, but not in this study, had either not been available throughout the 4-year period or did not qualify according to bone age. Comparisons will be made between these two studies throughout this paper, and will be designated as the "puberty" and "normative" studies, respectively, without further reference.

Night $l$ in the laboratory was the fifth day of the menstrual cycle for females (follicular phase). Subjects were required to abstain from medication and alcohol, and instructed not to nap. These restrictions were monitored daily by means of a presleep questionnaire administered just before bedtime.

The study encompassed 4 years, with each subject spending 3 consecutive nights in the laboratory each vear. Bedtime and arousal time conformed to their normal sleep habits. The first night was considered an adaptation night: therefore, first night data have been included in the analysis but were analyzed separately. Parameter averages for the second and third nights were obtained for each subject. and these were used in the subsequent analyses. The same subjects and identical procedures were employed during all 4 years of the study.

Electrode placements, recording techniques, and scoring procedures were the same as reported by Williams et al. (21), including $40 \mu \mathrm{V}$ peak to peak as the criterion for $\delta$ wave amplitude.

We were primarily concerned with determining the possibility of changes in sleep characteristics across the 4 years that subjects we re present in the study, and determining the equivalence of these chianges between sexes. Supplementary to the year to year changes, we also compared puberty subjects with the immediately preceding a)ge group (10-12 years) and the young adult age group (20-29 years) from the normative study. Secondary interest was to evaluate the first night effects for each of the 4 years.

Longitudinal comparisons for the 7 male and 10 female puberty su bjects were made using computer program BMDX 63 multivariatre general linear hypothesis (3). Specific questions addressed in this analysis of each sleep characteristic were the following. (I) Is there a consistent difference in sleep between males and females? (2) Do the longitudinal effects have a different pattern of change for males than for females? (3) Is there an initial laboratory night effect within years, and if so does it persist from year to year? (4) Are there systematic changes in sleep across the 4 years of the study irrespective of sex?

All of the above questions were tested with $F$ values derived from the Hotelling $T^{2}$ test in the multivariate analysis of variance procedure described by Morrison (15). Comparisons of results between the puberty subjects and previously reported normative data in younger and older age groups were made using $t$-tests for inciependent groups.

\section{RESULTS}

The analysis proceeded in a sequential manner. We first established that there were little or no indications of differences in sleep outcomes between the sexes, and that data for males and females could accordingly be combined. Secondly, we re-established the presence of adjustment effects associated with initial laboratory nights, justifying the eventual utilization of data from only the two later nights during all 4 years for each subject in evaluating all other effects.

\section{SEX DIFFERENCES}

There were no main effect differences due to sex, and only one parameter had a significant interaction between sex and years, i.e., total sleep time. Figure I illustrates that males had slightly more volatile changes than females from year to year, but with very little overall difference in total sleep time. As a consequence of the apparent lack of conclusive differences between sexes, all remaining analyses were made for the combined data without regard to sex. It may be noted that in the normative study a grand total of 4 statistically significant differences were found to be due to sex out of 100 comparisons within the $10-12,13-15,16-19$, and 20-29 year age groups. This rate could be expected to be due to chance alone. All of the females in age groups $13 \quad 15$ through menopause were studied during the follicular phase of their menstrual cycle, as were the females in this 4-year puberty study. Since hormonal differences may influence sleep, the test was run for all females in the follicular phase of the menstrual cycle as determined by basic daily temperature charts.

\section{FIRST NIGHT EFFECTS}

Table 1 illustrates the eight parameters exhibiting the most dramatic first night effects. The results indicate a disturbance of sleep on initial laboratory nights relative to later nights in all cases of statistical significance. Subjects had poorer sleep efficiency, more awakenings, fewer REM periods, more stage 0 , more stage 1 , less stage I-REM, longer sleep latency, and longer latency to stage $1-R E M$ on initial nights than on later nights. First night adaptation effects like these are well known to exist in sleep research when testing adult subjects in a study of short duration. Results reported here document clearly the persistence of these effects from year to year for subjects during their emergence from puberty. This is remarkable considering that all laboratory testing procedures and personnel remained constant throughout the period.

\section{YEAR TO YEAR EFFECTS}

Table 2 illustrates results of comparisons for all EEG parameters included in the analysis. The middle four columns of data represent outcomes for the 17 puberty subjects. Columns at the left and right represent data for 24 subjects ( 13 male and 11 female) and 20 subjects ( 10 male and 10 female), respectively, from the normative study. Statistical comparisons were $(l)$ between the age 10-12 normative group and year 1 for puberty subjects, (2) among the 4 years for puberty subjects, and (3) between year 4 for puberty subjects and the age 20-29 normative group.

For the most part, sleep characteristics during the 4 years immediately after onset of puberty appeared to represent a typical phase in the gradual patterns of changes across all ages. Generally, EEG parameters having marked age effects exhibited these effects during the 4 years of this study, and those parameters having either no effects or random fluctuations with age remained relatively constant for the puberty subjects. Our characterization of changes with age will be based on the ontogenetic trends described in the normative study.

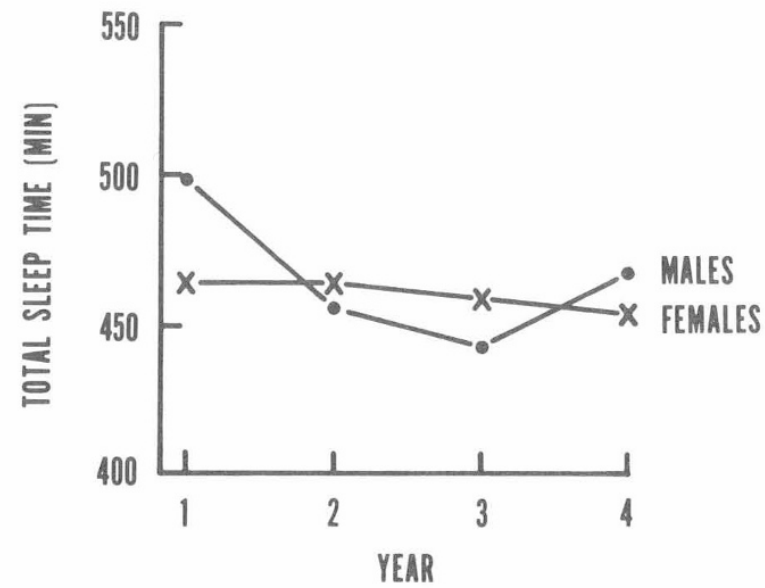

Fig. 1. Effect of age on total sleep time. 
Table 1. Effects of initial laboratory adjustment on selected sleep characteristics ${ }^{1}$

\begin{tabular}{|c|c|c|c|c|c|c|c|c|c|}
\hline \multirow[b]{2}{*}{ Parameter } & \multicolumn{2}{|c|}{ Yearl } & \multicolumn{2}{|c|}{ Year 2} & \multicolumn{2}{|c|}{ Year 3} & \multicolumn{2}{|c|}{ Year 4} & \multirow[b]{2}{*}{$\begin{array}{l}\text { All } \\
\text { year }\end{array}$} \\
\hline & $N t . l$ & $\begin{array}{l}\text { Nts. } \\
2 \& 3\end{array}$ & Nt.l & $\begin{array}{l}\text { Nts. } \\
2 \& 3\end{array}$ & $N t . l$ & $\begin{array}{l}\text { Nts. } \\
2 \& 3\end{array}$ & $N t . l$ & $\begin{array}{l}N t s . \\
2 \& 3\end{array}$ & \\
\hline Sleep efficiency $(\%)$ & $91.9^{2}$ & 96.3 & 94.8 & 95.9 & 94.3 & 95.7 & $92.4^{3}$ & 96.7 & 2 \\
\hline No. of awakenings & 3.3 & 2.2 & 4.6 & 2.7 & 1.9 & 1.1 & 2.4 & 1.4 & 3 \\
\hline No. of REM periods & $3.7^{3}$ & 4.4 & 4.4 & 4.3 & 4.3 & 4.7 & 4.2 & 4.7 & 2 \\
\hline$\%$ Stage 0 & 3.3 & 0.8 & 2.0 & 0.8 & 0.8 & 0.7 & 2.6 & 0.9 & 3 \\
\hline$\%$ Stage 1 & $4.6^{3}$ & 3.2 & 3.6 & 2.9 & $4.6^{3}$ & 3.4 & 5.8 & 3.1 & 2 \\
\hline \% Stage I-REM & $21.7^{2}$ & 25.6 & 25.2 & 26.0 & $24.8^{2}$ & 27.5 & $23.8^{2}$ & 27.6 & 4 \\
\hline Sleep latency $(\mathrm{min})$ & 44 & 13 & 15 & 15 & 24 & 17 & $18^{3}$ & 11 & 3 \\
\hline Latency to stage $1-R E M(\mathrm{~min})$ & $164^{2}$ & 122 & 127 & 124 & 105 & 96 & $143^{3}$ & 94 & 4 \\
\hline
\end{tabular}

${ }^{1} \mathrm{Nt}$.: night; REM: rapid eye movement.

${ }^{2}$ Significant at the $1 \%$ level.

${ }^{3}$ Significant at the $5 \%$ level.

${ }^{4}$ Significant at the $0.1 \%$ level.

Table 2. Longitudinal data for puberty subjects in comparison with younger and older age groups ${ }^{1}$

\begin{tabular}{|c|c|c|c|c|c|c|c|c|c|}
\hline \multirow[b]{2}{*}{ EEG parameter } & \multirow[b]{2}{*}{$\begin{array}{l}\text { Normative } \\
\text { age } 10-12\end{array}$} & \multirow[b]{2}{*}{$\begin{array}{l}\text { Signif- } \\
\text { icance }\end{array}$} & \multicolumn{5}{|c|}{ Longitudinal puberty subjects } & \multirow[b]{2}{*}{$\begin{array}{l}\text { Signif- } \\
\text { icance }\end{array}$} & \multirow[b]{2}{*}{$\begin{array}{c}\text { Normative } \\
\text { age } 20-29\end{array}$} \\
\hline & & & Year 1 & Year 2 & $\begin{array}{l}\text { Signif- } \\
\text { icance }\end{array}$ & Year 3 & Year 4 & & \\
\hline Total sleep time (min) & $560 \pm 27$ & 2 & $478 \pm 28$ & $460 \pm 18$ & 3 & $452 \pm 19$ & $458 \pm 44$ & 2 & $424 \pm 19$ \\
\hline Sleep efficiency $(\%)$ & $95.0 \pm 2.5$ & & $96.3 \pm 1.7$ & $95.9 \pm 2.6$ & & $95.7 \pm 2.4$ & $96.7 \pm 3.3$ & & $95.5 \pm 3.2$ \\
\hline \multicolumn{10}{|l|}{ Number of } \\
\hline Awakenings & $1.4 \pm 1.2$ & & $2.2 \pm 2.2$ & $2.7 \pm 2.0$ & 3 & $1.1 \pm 1.0$ & $1.4 \pm 1.5$ & & $2.1 \pm 2.1$ \\
\hline Stage shifts & $35 \pm 9$ & & $39 \pm 8$ & $39 \pm 10$ & & $36 \pm 8$ & $36 \pm 9$ & & $37 \pm 9$ \\
\hline REM periods & $5.2 \pm 0.8$ & 2 & $4.4 \pm 1.0$ & $4.3 \pm 0.9$ & & $4.7 \pm 0.7$ & $4.7 \pm 0.8$ & 4 & $4.1 \pm 0.8$ \\
\hline \multicolumn{10}{|l|}{ Percentage of } \\
\hline Stage 0 & $1.4 \pm 1.9$ & & $0.9 \pm 0.9$ & $0.8 \pm 0.7$ & & $0.7 \pm 0.8$ & $0.9 \pm 1.8$ & & $0.9 \pm 0.9$ \\
\hline Stage 1 & $3.0 \pm 2.0$ & & $3.2 \pm 1.7$ & $2.9 \pm 1.1$ & & $3.4 \pm 1.1$ & $3.1 \pm 0.5$ & 4 & $4.3 \pm 2.0$ \\
\hline Stage l-REM & $26.9 \pm 2.6$ & & $25.5 \pm 4.1$ & $26.0 \pm 5.0$ & & $27.5 \pm 3.8$ & $27.6 \pm 4.3$ & & $26.7 \pm 4.9$ \\
\hline Stage 2 & $47.6 \pm 6.0$ & & $47.9 \pm 6.3$ & $44.9 \pm 6.2$ & & $46.8 \pm 5.0$ & $49.4 \pm 6.6$ & & $48.8 \pm 6.4$ \\
\hline Stages 3 and 4 & $21.0 \pm 4.0$ & & $22.5 \pm 2.8$ & $25.3 \pm 6.2$ & 3 & $21.7 \pm 4.2$ & $19.1 \pm 4.4$ & & $19.3 \pm 5.8$ \\
\hline \multicolumn{10}{|l|}{ Latency in minutes to } \\
\hline Sleep onset & $17 \pm 10$ & & $13 \pm 7$ & $15 \pm 13$ & & $17 \pm 10$ & $11 \pm 13$ & & $14 \pm 10$ \\
\hline Stage 0 & $374 \pm 146$ & & $290 \pm 124$ & $250 \pm 83$ & & $288 \pm 158$ & $344 \pm 113$ & 4 & $231 \pm 1465$ \\
\hline Stage I-REM & $148 \pm 32$ & 3 & $122 \pm 38$ & $124 \pm 35$ & 3 & $96 \pm 36$ & $94 \pm 29$ & & $94 \pm 34$ \\
\hline Stage 2 & $7 \pm 4$ & & $5 \pm 2$ & $5 \pm 2$ & & $6 \pm 2$ & $6 \pm 2$ & & $8 \pm 4$ \\
\hline Stage 3 & $15 \pm 4$ & & $14 \pm 2$ & $14 \pm 3$ & & $16 \pm 2$ & $17 \pm 4$ & 3 & $22 \pm 8$ \\
\hline Stage 4 & $18 \pm 4$ & & $18 \pm 2$ & $18 \pm 5$ & & $20 \pm 3$ & $20 \pm 4$ & 4 & $27 \pm 10$ \\
\hline
\end{tabular}

${ }^{1}$ EEG: electroencephalograph; REM; rapid eye movement.

${ }^{2}$ Significant at the $0.1 \%$ level.

${ }^{3}$ Significant at the $5 \%$ level.

${ }^{4}$ Significant at the $1 \%$ level.

Total sleep time decreases markedly for the span of years reported in Table 2. The puberty subjects had trends falling right in line with the normal ontogenetic patterns, having less sleep time than subjects in the $10 \quad 12$ year age range, decreasing their sleep time during the 4-year period, but having longer sleep time than subjects in the 2029 year age range. Despite their decrease in total sleep time, subjects maintained constant levels of sleep efficiency. This important result suggests that individuals may normally have decreasing needs for sleep with increasing age (through age range 30-39) and fulfill their needs to the same extent.

Like sleep efficiency, individuals normally have the same number of awakenings per night until sleep becomes more disturbed during middle age and beyond. Puberty subjects had slightly more awakenings on the average in the first 2 years than in the last 2 years. This could possibly represent a disturbance during initial puberty years. The number of sleep stage shifts during the night varies around a constant mean value throughout all ages.
Puberty subjects were right in line with normative data. On the other hand, the number of REM periods during the night decreases sharply for individuals from childhood through adolescence. remaining constant thereafter. Puberty subjects may have gone somewhat contrary to the pattern, but nonetheless remained at levels intermediate to younger and older age groups.

Percentages of the various sleep stages are fairly constant for individuals from age range 10-12 through age range 30-39. During this span of time, individuals have slightly increasing amounts of light sleep (stages $l$ and 2 ) and correspondingly decreasing amounts of deep or slow wave sleep (stages 3 and 4 ). No consistent pattern of change seems to occur for stage l-REM, the so-called dream stage. As Table 2 indicates, our puberty subjects had percentage profiles in nearly perfect agreement with the normal ontogenetic process. We may wonder about the peaking of slow wave sleep during the second year at a level higher than any other age group. Actually the normative data suggest that slow wave 
sleep reaches a peak at some point during the teen years. Our data for puberty subjects suggest that this peak occurs around the second year of puberty.

From normative data, it was shown that latencies to both stages 3 and 4 have increasing trends with age, reflecting a decreasing total amount of these stages. Similarly, latency to stage 0 (first awakening) decreases continuously with advancing age, especially from middle age onward. Except for the reversion between ages 13-15 (too short) and 16-19 (too long), latency to stage l-REM also has decreasing trends with age. Latency to stage 2 remains at a constant low level, and latency to sleep onset decreases somewhat after the teen years. The data for puberty subjects agreed with the normal ontogenetic patterns to a large extent. Latencies to stages 3 and 4 both increased slightly during the 4 years in full agreement with the normative trends. Latency to stage I-REM exhibited decreasing values in the last 2 years, in essential agreement with normative data. Furthermore, latency to sleep onset and latency to stage 2 both remained relatively constant, agreeing with normative data. The only possible exception was latency to stage 0 which had its lowest mean value in year 2 and highest mean value in vear 4. whereas the normative data would have predicted steadily decreasing values (earlier awakenings) with increasing age.

\section{DISCUSSION}

We found that female subjects studied during the follicular phase of their menstrual cycle had sleep EEG characteristics almost indistinguishable from those of male subjects. In comparison to other age groups (21), the same results were obtained not only for the 4 years immediately after puberty, but extending at least to middle age. The puberty subjects confirmed previously held conclusions that individuals generally require a laboratory adaptation night each time they return after a period of absence: first night effects consistently showed disturbance of sleep relative to succeeding nights.

Comparing sleep characteristics among the 4 years that the puberty subjects were studied with previously published normative data for groups of subjects spanning the ages of our sample, we found very few indications distinguishing puberty as unique. Phenomena were observed, especially during the second year, that may combine to describe an unstable period fraught with conflicting demands. In one sense, the subjects' sleep was disturbed, having the greatest average number of awakenings and shortest latency to first awakening. In another sense, they experienced an apparent need for deep sleep, having the highest percentage of stages 3 and 4 and lowest percentage of stage 1 . These two results perhaps reflect the labile nature of the period of puberty.

The fact that stages 3 and 4 were increased during the second year of puberty may have biologic import. Slow wave sleep may be the backdrop for the physiologic changes observed during puberty. Secretion of growth hormone $(\mathrm{GH})$ in particular may reflect the changes seen in the percentage of slow wave sleep. Episodic secretion of $\mathrm{GH}$ is generally associated with the occurrence of slow wave sleep in adults $(8,16,19)$, but much more needs to be learned about its physiologic significance. Finkelstein et al. (1972) have studied 24-hr secretion of growth hormone in normal prepubertal, pubertal, and adult subjects. GH secretion in prepubertal children was manifest only during sleep, whereas in both pubertal and adult individuals episodic secretion of $\mathrm{GH}$ occurred during the day as well as during sleep, with total 24-hr GH secretion decreasing with age. However, the relationship between $\mathrm{GH}$ secretion and sleep stage percentages was not examined.

$\mathrm{GH}$ secretion should be further examined during puberty to ascertain whether it would parallel the occurrence of slow wave sleep, and to ascertain the relation of GH to other psychobiologic changes. We would expect to see an elevation of $\mathrm{GH}$ secretion during puberty, in accordance with the elevation of slow wave sleep.

A longitudinal study (13) of pubertal males has shown that serum luteinizing hormone levels increase throughout puberty, whereas the level of testosterone progressively increased from before the appearance of sexual hair until the adult hair distribution was completed. Further, not only the amount of hormonal secretion but also its time of occurrence relative to the secretion of other hormones and the occurrence of other physiologic events appear to be an integral part of the pubertal mechanism. The initial increase in luteinizing hormone concentration occurs before the level of testosterone concentration is appreciable. Levels of testosterone and follicle-stimulating hormone rise only after genital growth, although continuation of genital growth occurs even after testosterone elevation is no longer detectable (13). Also, Feinberg (6) has proposed that metabolic activity and neuronal integrity may be intimately connected with sleep patterns. Feinberg (5) has shown a general relationship of decreasing metabolism with increasing age. Thus, it would follow that there should be differences in the sleep patterns of prepubertal, pubertal, and young adult groups. Our results, however, do not support such a contention. The generally stabilized sleep patterns observed during puberty in conjunction with the well known intensive physiologic and psychologic changes characteristic of this period may seem incongruous. Congruity may lie in the relationship of slow wave sleep to other psychobiologic changes occurring during this time.

Whether puberty represents an integrated sequence of developments in a positive feedback loop or whether it is governed by a master process has not been determined. In any case, the simultaneous changes occurring in brain and peripheral organs would make it a logical candidate for changes in sleep. However. as the results of this study suggest, the sleep characteristics of this group are surprisingly resistant to substantive changes. Not only were there no sleep disturbances, but the significant changes observed were of a positive nature, i.e., those parameters of sleep that we normally consider of importance were maximized as indicated by increase in stages 3 and 4 . The very significant differences observed in the sleep patterns across the 4 years of study mitigate against any notion that the mechanisms governing sleep are vulnerable to disruption during puberty. Rather, the sleeping brain seems to be organized to insure stability of sleep. This, of course, could be part of a survival system. If so, changes in this system should be considered a serious problem. It is important to emphasize that the tests with females were run during the follicular phase of their menstrual cycles, a time during which the hormonal difference between male and female is minimized, even though differences in hormonal concentration between male and female are still appreciable. It is reasonable to suspect that, if other periods of the female cycle were chosen, there may have been sex differences in the sleep characteristics. This might prove to be an interesting area for further investigation.

\section{SUMMARY}

Longitudinal comparison of parameters of sleep in pubertal males and females across 4 years of electrophysiologic recording revealed the following characteristics. Essentially, there were no sex differences in the sleep of pubertal males or females. The parameters of sleep were surprisingly resistant to change across the 4 years, with sleep efficiency maintaining constant levels. Puberty subjects continued the pattern seen across all ages, particularly evident in the significant decreases in total sleep time and latency to stage $1-R E M$ across the 4 years. Exceptions to this were the significant increases in the number of awakenings and the percentages of slow wave sleep observed during the second year of puberty. Finally, there was persistent evidence of first night effects each year across the 4-year evaluation.

\section{REFERENCES AND NOTES}

1. Adey, W., Kado, R., and Walter, D.: Computer analysis of EEG data from Gemini flight GT-7. Aerospace Med. 38: 345 (1967).

2. Boyar, R., Finkelstein, J., Roffwarg, H., Kapen, S., Weitzman, E., and Hellman, L.: Synchronization of augmented luteinizing hormone secretion with sleep during puberty. N. Engl. J. Med., 287: 582 (1972).

3. Dixon W. J. (editor): BMD Biomedical Computer Programs X-series Supple- 
ment (University of California Press, Berkeley, 1972).

4. Donovan, B.. and Van Der Werff Ten Bosch, J.: Physiology of Puberty. (The Williams \& Wilkins Co., Baltimore, 1965).

5. Feinberg, I.. and Carlson. V. R.: Sleep variables as a function of age in man. Arch. Gen. Psychiat. 18:239 (1968).

6. Feinberg, I., Koresko, R. L., and Heller, N. EEG sleep patterns as a function of normal and pathological aging in man. J. Psychiat. Res., 5: 107 (1967).

7. Hartmann, E.: The Functions of Sleep (Yale University Press, New Haven, 1973).

8. Honda, Y.. Takahashi, K.. Takahashi, S.. Azumi, K.. Irie, M.. Sakuma, M.. Tsushima, T., and Shizume, K.: Growth hormone secretion during nocturnal sleep in normal subjects. J. Clin. Endocrinol. Metab.. 29: 20 (1969).

9. Johnson, L. C.: Psychological and physiological changes following total sleep deprivation. In: A. Kales: Sleep Physiology and Pathology: A Symposium, pp. 206-220 (J. B. Lippincott Company, Philadelphia, 1969).

10. Kleitman, N.: Sleep and Wakefulness, Revised Edition (University of Chicago Press, Chicago, 1963).

11. Koella, W: The physiology and pharmacology of sleep. In: J. Mendels: Biological Psychiatry. pp. 263-296 (John Wiley \& Sons, New York. 1973).

12. Kramer, M.: Dream Psychology and the New Biology of Dreaming (Charles C Thomas, Springfield, III., 1969).

13. Lee, P. A., Jaffe. R. B., Midgley, A. R., Jr.: Serum gonadotropin, testosterone and prolactin concentrations throughout puberty in boy: A longitudinal study. J. Clin. Endocrinol. Metab., 39: 664 (1974)

14. Lester, B., Burch, N., and Dossett, R.: Nocturnal EEG-GSR profiles: The influence of presleep states. Psychophysiology, 3: 238 (1967).

15. Morrison, D. F.: Multivariate Statistical Methods (McGraw-Hill, New York.
1967).

6. Parker, D. C Sassin J F. Mace, J, W Gotlin, R, W and Rossman, L. G. Human growth hormone release during sleep: Electroencephalographic correlation. J. Clin. Endocrinol. Metab., 29:871 (1969).

17. Roffwarg, H. P.. Muzio, J. N.. and Dement, W. C.: Ontogenetic development of the human sleep-dream cycle. Science, 152:602 (1966).

18. Shurley, J., Pierce. C., Natani, K.. and Brook, R.: Sleep activity patterns at south pole station: A preliminary report. Arch. Gen. Psychiat. 22: 385 (1970).

19. Takahashi, Y.. Kipnis, D. M.. and Daughaday, W. H.: Growth hormone secretion during sleep, J. Clin. Invest. 47:2079(1968).

20. Webb. W.: Sleep deprivation: Total, partial and selective. In: M. H. Chase: Perspectives in the Brain Sciences, Vol. 1. The Sleeping Brain, pp. 323361 (Brain Information Service/Brain Research Institute. UCLA. Los Angeles, 1972)

21. Williams, R. L.. Karacan, I., Hursch, C. J.: Electroencephalography (EEG) of Human Sleep: Clinical Applications (John Wiley \& Sons, New York. 1974).

22. Williams, R. L.. Karacan, I.. Hursch, C. J... and Davis, C. E.: Sleep patterns of pubertal males. Pediat. Res. 6: 643 (1972).

23. Bone age determinations were made by Alvin H. Felman. M.D.. Department of Radiology, College of Medicine, University of Florida, Gainesville, Fla

24. This work was supported in part by Grant MH-15508 from the National Institute of Mental Health and Gainesville and Houston Veterans Administration Hospitals research funds.

25. Requests for reprints should be addressed to: I. Karacan, M.D.. Department of Psychiatry, Baylor College of Medicine, Texas Medical Center. Houston. Tex. 77025 (USA).

26. Accepted for publication July 10, 1975

\title{
Effect of Sulfadimethoxine on Tissue Distribution of $\left[{ }^{14} \mathrm{C}\right]$ Bilirubin in the Newborn and Adult Hyperbilirubinemic Gunn Rat
}

\author{
DAVID R. DAVIS (29) AND ROGER A. YEARY
}

Department of Veterinary Physiology and Pharmacology, The Ohio State University, Columbus, Ohio, USA

\section{Extract}

Sulfadimethoxine significantly reduced plasma bilirubin levels and altered the tissue bilirubin distribution in both the newborn and the adult Gunn rat. The majority of the unbound bilirubin appeared to distribute preferentially to the intestine and liver in the newborn, whereas in the adult the unbound bilirubin was taken up primarily by the liver. The bilirubin content of brain tissue from both age groups was significantly higher after sulfadimethoxine treatment.

\section{Speculation}

A decrease in the available extravascular bilirubin binding sites, indicated by an increased concentration of bilirubin in the carcass and an apparent decreased capacity of the liver of the newborn Gunn rat to bind bilirubin, would suggest that kernicterus in the Gunn rat neonate is associated with increased bilirubin concentrations in the brain. However, the similar uptake of bilirubin by the brain tissue of both the newborn and the adult Gun rat raises the question of increased sensitivity of the newborn brain to bilirubin toxicity.

Sulfonamide therapy in hyperbilirubinemic newborn infants has resulted in a significant increase in the incidence of kernic- terus accompanied by a decrease in serum bilirubin levels (21). Johnson (9) reported a similar syndrome for the young hyperbilirubinemic Gunn rat. The significance of the decrease in plasma bilirubin levels during sulfonamide therapy was not recognized until Odell $(16,17)$ proposed that certain anions including sulfonamides were capable of displacing protein-bound bilirubin from albumin. This concept stimulated widespread interest in the competitive protein binding of anions and shifted the clinical interests from total plasma bilirubin levels to free or unbound plasma bilirubin $(5,10,14)$.

Since it is the unbound bilirubin which freely diffuses into extravascular compartments such as neural tissue $(2,20)$, where its more serious toxic effects may be manifest, it is logical to examine the tissue distribution of unbound bilirubin in the presence of a drug that can displace bilirubin from serum albumin at therapeutic serum levels. Furthermore, since kernicterus is a comparatively rare phenomenon in hyperbilirubinemic adults and there is disagreement on age-related permeability of the bloodbrain barrier to bilirubin, comparable studies in newborn and adult animals were undertaken. For this purpose the Gunn rat was chosen as the animal model. We have found (23) sulfadimethoxine to be significantly more toxic when injected into the 2-day-old icteric Gunn rat $\left(\mathrm{LD}_{50}=63 \mathrm{mg} / \mathrm{kg}, 95 \%\right.$ confidence 